\title{
Circuit
}

Musiques contemporaines

\section{Analyse électroacoustique}

\section{Vincent Tiffon}

Volume 17, numéro 3, 2007

Musique in situ

URI : https://id.erudit.org/iderudit/017597ar

DOI : https://doi.org/10.7202/017597ar

Aller au sommaire du numéro

Éditeur(s)

Les Presses de l'Université de Montréal

ISSN

1183-1693 (imprimé)

1488-9692 (numérique)

Découvrir la revue

Citer ce compte rendu

Tiffon, V. (2007). Compte rendu de [Analyse électroacoustique]. Circuit, 17(3),

119-123. https://doi.org/10.7202/017597ar

Ce document est protégé par la loi sur le droit d'auteur. L'utilisation des services d'Érudit (y compris la reproduction) est assujettie à sa politique d'utilisation que vous pouvez consulter en ligne.

https://apropos.erudit.org/fr/usagers/politique-dutilisation/
Cet article est diffusé et préservé par Érudit.

Érudit est un consortium interuniversitaire sans but lucratif composé de l'Université de Montréal, l'Université Laval et l'Université du Québec à Montréal. Il a pour mission la promotion et la valorisation de la recherche. https://www.erudit.org/fr/ 
ACTUALITÉs

\section{Analyse électroacoustique}

Vincent Tiffon

Simoni, Mary (dir.), Analytic Methods of Electroacoustic Music, New York, Taylor \& Francis Group, 2006.

\section{Prolégomènes}

Il est bienvenu d'entamer un ouvrage dont le sujet traite des méthodes analytiques de la musique électroacoustique - catégorie qui inclut les musiques mixtes, dans la nomenclature anglo-saxonne - en privilégiant la mise en perspective du rapport œil/oreille dans la création musicale. La description des modes de représentation du musical et des vertiges de la représentation des abstractions musicales (page 3 et suivantes) permet à Mary Simoni, directrice de l'ouvrage, de proposer en introduction une lecture qui pourrait être qualifiée de médiologique.

En mettant en avant les spécificités propres aux différents types de représentation de la musique, allant des transcriptions-traductions (d'un langage à l'autre) aux représentations les plus symboliques, en passant par les transcriptions littéraires ou encore la représentation classique en quatre paramètres hauteur/intensité/durée/timbre, Mary Simoni met l'accent sur les avantages de la représentation sonagraphique pour la restitution indicielle des musiques cultuelles (chant grégorien, musiques de tradition orale...) comme des musiques symboliques (musique écrite de la Renaissance à aujourd'hui) ou encore des sons de synthèse issus des calculs des programmes musicaux (avec l'exemple de Csound). Le chapitre consacré à l'influence de la représentation en musique (p. 8) met en perspective le principe même de la notation en musique. Pour revenir au cadre plus étroit de la musique électroacoustique, Mary Simoni défend la pratique d'une lecture plurielle de telles musiques 
grâce aux représentations graphiques plurielles: l'analyse qu'elle propose dans le corpus d'analyses qui suit son introduction reflète cette préoccupation. Ce faisant, cet article introductif, prélude au recueil d'analyse, éclaire la philosophie générale de l'ouvrage. Il vise en outre une certaine exhaustivité, objectif qui ne peut être atteint dans le cadre d'un ouvrage de 300 pages - l'absence de déterminant dans le titre Analytical Methods suggère que l'exhaustivité des méthodes analytiques n'est évidemment pas un objectif avoué. Cette introduction en forme de quasi-manifeste est judicieusement prolongée par la description fine que donne Norman Adams des procédés techniques utilisés pour transposer l'analyse d'un signal sonore dans le domaine de la représentation graphique. L'analyse critique des transcriptions les unes par rapport aux autres permet à Norman Adams de poser en tête de l'ouvrage - qu'il convient à ce titre de lire de manière linéaire - les bases réflexives indispensables à la compréhension des relations oil/oreille: la question de l'instrumentation de l'oreille par l'œil au cœur de la pensée graphosphérique des musiques «savantes» occidentales se pose de nouveau dans le cadre du travail analytique de la musique «fixée sur support électronique».

\section{Qualités éditoriales}

Cet ouvrage collectif présente de nombreuses qualités éditoriales, qui vont de la composition plurielle en matière de diversité des œuvres analysées à la richesse des annexes. L'ouvrage par ailleurs s'inscrit dans une collection éditoriale exigeante Studies on New Music Research, dirigée par Marc Leman, de l'IPEM de Gand (Belgique). L'ouvrage collectif, fruit de huit auteurs, est composé d'une introduction (mentionnée précédemment), à laquelle s'ajoutent un article de réflexion plus globale (Visualization of Musical Signals de Norman Adams), un compte rendu détaillé d'expériences d'écoute sur deux pièces électroacoustiques, suivi de six analyses d'œuvres électroacoustiques ou mixtes. Ce corpus de textes s'achève sur un appareillage critique non négligeable constitué d'une bibliographie commentée, d'un glossaire des termes musicaux, techniques et scientifiques, d'un index général (œuvres, compositeurs, termes) et enfin d'un CD-Rom.

L'appareil critique de l'ouvrage est soigné. La bibliographie générale (indépendante des bibliographies propres à chaque analyse) qui traite de la musique électroacoustique dans ses généralités et des méthodes analytiques est une bibliographie commentée. Elle n'est cependant pas exhaustive. En revanche, un glossaire présentant des entrées incontestablement utiles sur des notions parfois complexes accompagne cette compilation d'articles. Un index des termes techniques, un index général (compositeurs, œuvres, 
notions techniques et musicales...) ainsi qu'un résumé biographique des auteurs complètent l'ouvrage. Enfin, le CD-Rom fourni est un complément indispensable à ce genre d'entreprise éditoriale, même si l'on regrettera l'absence de compatibilité Mac/PC de certains exemples, l'accès peu aisé aux extraits vidéo et le peu d'interactivité offerte, à l'exception notable des exercices interactifs proposés par Michael Clarke pour mieux comprendre les présupposés acoustiques de l'œuvre analysée par ses soins. D'ailleurs, Michael Clarke, par cette utilisation adéquate du multimédia, réintroduit la dimension de la participation esthétique, clé de l'expérience esthétique. Enfin, signalons l'absence regrettable, dans ce CD-Rom, des reproductions audio des œuvres ou de certains extraits d'œuvres qu'il est aujourd'hui difficile de se procurer (à l'exception notable de Riverrun de Barry Truax qui est reproduite in extenso). De même, l'absence de synchronisation entre les quelques extraits sonores et la partition, le sonagramme ou les lignes de codes informatiques correspondantes, peut apparaître comme un défaut majeur.

Sur le corpus de textes, l'éclectisme est de rigueur. Les œuvres analysées relèvent à la fois du répertoire des arts des sons fixés sur support et des musiques mixtes, conformément à l'acception anglo-saxonne du terme «musique électroacoustique». Les «arts des sons fixés sur support» sont ici représentés par Valley Flow (1991-1992) de Denis Smalley, Prochaine station (1990) de Christian Calon et Claude Schryer, Mortuos plango, vivos voco... (1980) de Jonathan Harvey, I am sitting in a room (1969) d'Alvin Lucier et Riverrun (1986) de Barry Truax. Les musiques mixtes analysées sont As If (1982) de Paul Lansky pour quatuor à cordes et bande magnétique - œuvre dont l'effectif instrumental et les matériaux correspondent à une configuration très fréquente du répertoire mixte, à savoir celle du dialogue de l'instrument et de son double électronique - et deux pièces de Philippe Manoury associant des instruments acoustiques et des sons électroniques générés ou déclenchés en temps réel, Jupiter (1982) pour flûte et électronique en temps réel, et l'opéra K... (2001). Manque cependant une référence aux œuvres pionnières du répertoire électroacoustique et mixte. L’ancrage majoritaire sur les œuvres des années 1980 (quatre analyses sur les huit proposées) rappelle le niveau de productivité élevé des compositeurs de ces années. L'absence d'œuvres majeures des années 1960 et 1970 est compensée, selon nous, par la présence de trois œuvres de référence que sont Mortuos plango, vivos voco, Jupiter et, Riverrun). Signalons enfin la sur-représentation des compositeurs anglophones ou francophones. Cet éclectisme reflète des esthétiques ou des approches compositionnelles relativement larges au regard d'un corpus si restreint: œuvres conceptuelles (I am sitting in a room, et dans une moindre 
1. Stéphane Roy, L'analyse des musiques électroacoustiques: modèles et propositions, Paris, l'Harmattan, 2003 mesure Prochaine station qui peut relever du «cinéma pour l'oreille»), musique acousmatique (Valley Flow), musique électronique (Riverrun), musique électroacoustique de l'esthétique spectrale (Mortuos plango, vivos voco...), musique valorisant le prima de l'écriture graphique (Jupiter, K., As If).

\section{Méthodes analytiques plurielles}

L'ouvrage se propose d'éclairer la problématique de l'analyse de la musique électroacoustique par la présentation et la mise en perspective de méthodes analytiques plurielles. Là où Stéphane Roy présentait les différentes méthodes analytiques en privilégiant son seul point de vue de compositeur et d'analyste - ce qui n'altère en rien la qualité scientifique du projet, plus encore lui donne une cohérence -, un livre collectif comme Analytical Methods of Electracoustic Music offre, par essence, une exposition des différentes orientations analytiques, au risque de perdre précisément une certaine cohérence. Le lecteur est invité à construire cette cohérence, en faisant dialoguer à distance les œuvres et les méthodes présentées par des universitaires de langue anglaise, le plus souvent compositeurs, toujours selon la tradition anglosaxonne des départements de musique et musicologie.

L'ouvrage présente en réalité deux articles (dont l'introduction de Mary Simoni) qui s'interrogent sur les méthodes. Les sept autres articles proposent des comptes rendus d'analyse, selon des orientations méthodologiques parfois différentes, d'où le titre général de l'ouvrage: Méthodes analytiques de la musique électroacoustique. Le plus souvent, les analyses présentent de manière linéaire une description interprétative de l'œuvre chronologique, mouvement par mouvement lorsque l'œuvre s'y prête. Cette linéarité n'exclut pas la présence de quelques paragraphes plus transversaux, mettant en scène les thèses interprétatives que l'auteur souhaite défendre (Mickael Clarke, Andrew May, Benjamin Broening, Mary Simoni). Ce type de valorisation de l'analyse est compatible avec le recours aux méthodes analytiques revendiquées. Mary Simoni est l'auteure qui propose l'analyse mettant en jeu le plus de méthodes simultanées (dont la Set Theory) - en raison notamment de la catégorie mixte de l'œuvre analysée. Cette pluralité de méthodes pour la même analyse favorise une lecture très fouillée de l'œuvre. À l'évidence, la catégorie à laquelle appartiennent les œuvres analysées et les technologies utilisées pour leur élaboration conditionne les orientations analytiques et le choix des outils d'analyse. Pour ne citer que ces deux exemples, les technologies de synthèse/resynthèse ouvrent sur l'explication de l'esthétique spectrale de Harvey dans Mortuos plango, vivos voco..., alors que les technologies de traitement du signal en temps réel imposent l'exploration des questions 
de l'interactivité et de la place primordiale de l'interprète. Ces évidences n'excluent pas le recours aux méthodes analytiques à caractère paradigmatique, avec occasionnellement des «mises en tableau» pour aboutir in fine à des interprétations d'ordre esthétique. Mais le plus souvent, des contraintes externes influent sur le résultat (absence ou non des données poïétiques du compositeur, mise à disposition ou non des données des programmes informatiques...). Des analyses très documentées (comme celles d'Andrew May, Michael Clarke, Mara Helmuth, Momilani Ramstrum) donnent lieu à des textes, certes parfois de lecture difficile, mais dont la richesse des données est indispensable à une lecture exhaustive de l'œuvre. Parallèlement aux reproductions sur le CD-Rom de partitions, sonagrammes ou encore MSD (Music Structure Discovery) réunis par exemple dans l'analyse de K... de Manoury, les textes proposent une documentation iconographique, il est vrai, parfois peu lisible (comme pour certains sonagrammes) en raison du format $\mathrm{A}_{5}$. Cette faiblesse est partiellement compensée par la lecture avec agrandissement d'écran du CD-Rom.

Des questions difficiles comme celle de l'analyse de l'espace interne (de la composition) mais aussi de l'espace externe (de la diffusion - avec ou sans interprétation) des œuvres sont abordées non sans rigueur, notamment pour la pièce Riverrun (pour l'espace interne, composé en 8 pistes) et l'opéra K... (pour l'espace externe). Ainsi, quand la plupart des publications (y compris des publications multimédias) font le choix d'éluder de telles difficultés, les auteurs de cet ouvrage s'y frottent en se livrant à des analyses techniques exigeantes et nous offrent des conclusions d'ordre esthétique probantes, comme c'est le cas pour Momilani Ramstrum pour l'opéra K... 